\title{
Article \\ A Study on a Prediction Model of E-Bike Expansion Degree at Irregular Signalized Intersections
}

\author{
Ting Tan, Jianxiao Ma*, Zhen Yang, Mengyue Zhu, Chenhong Zong and Hao Li \\ College of Automobile and Traffic Engineering, Nanjing Forestry University, Nanjing 210037, China; \\ tanting@njfu.edu.cn (T.T.); zyang@njfu.edu.cn (Z.Y.); zhumengyue@njfu.edu.cn (M.Z.); \\ zongch@njfu.edu.cn (C.Z.); 171604307@njfu.edu.cn (H.L.) \\ * Correspondence: majx@njfu.edu.cn; Tel.: +86-025-8542-7652
}

check for updates

Citation: Tan, T.; Ma, J.; Yang, Z.; Zhu, M.; Zong, C.; Li, H. A Study on a Prediction Model of E-Bike Expansion Degree at Irregular Signalized Intersections. Appl. Sci. 2021, 11, 6852. https://doi.org/ 10.3390/app11156852

Academic Editor: Luís Picado Santos

Received: 20 June 2021

Accepted: 24 July 2021

Published: 26 July 2021

Publisher's Note: MDPI stays neutral with regard to jurisdictional claims in published maps and institutional affiliations.

Copyright: (c) 2021 by the authors. Licensee MDPI, Basel, Switzerland. This article is an open access article distributed under the terms and conditions of the Creative Commons Attribution (CC BY) license (https:/ / creativecommons.org/licenses/by/ $4.0 /)$.
Abstract: The deviations of straight-going traffic at irregular signalized intersections lead to obvious expansion characteristics of e-bikes. This situation increases the possibility of collisions between motor vehicles and e-bikes. In order to study the change of expansion degree of straight-going e-bike at irregular signalized intersections, the video trajectory extraction technology is used to obtain relevant data of e-bikes during green light release periods at irregular signalized intersections. In addition, we combined the flow and spacing characteristics of e-bikes and used a clustering method to analyze the release stage and release groups. Therefore, the Group 1 of e-bikes in the early green light release was determined to be the main research object of expansion degree. According to the static and dynamic factors, a prediction model for the expansion degree of straight-going e-bikes at irregular signalized intersections was established based on the beetle antennae search-back propagation (BASBP) neural network model. Finally, the evaluation indexes were compared and analyzed before and after the beetle antennae search (BAS) algorithm optimization. The results showed that the BAS-BP neural network prediction model was better than that of the back propagation (BP) neural network. The results could provide a theoretical reference for improving the efficiency of mixed traffic flow at irregular signalized intersections.

Keywords: traffic safety; e-bike; irregular signalized intersection; expansion degree; BAS-BP neural network

\section{Introduction}

In recent years, there have been an increasing number of e-bikes on the urban roads. As of 2019, the global market value of e-bikes reached 57.5 billion yuan (7.475 billion euros) and is expected to grow to 73.9 billion yuan (9.607 billion euros) in 2026. Among them, China has more than 300 million e-bikes, ranking first in the world [1]. Due to the popularity of e-bikes, traffic problems in cities are complex and the operation efficiency and safety of intersections are facing challenges. In Poland, an adaptive signal control method was adopted to adjust the length of vehicle queue [2]. In China, the number of public transportation vehicles per 10,000 persons in the city is positively associated with commuting by non-motor transport (by walking or cycling) [3]. Moreover, contraflow left-turn lane (which dynamically uses a portion of the opposing through lanes as additional left-turn lanes) was used to meet the demand of left-turns [4]. However, most of the optimization strategies are only for motor vehicle flow. The purpose of intersection signal control is to separate the traffic conflicts in the time distribution, but it cannot completely solve the traffic conflicts in the space distribution. Tang et al. [5] showed that the rate of accidents involving e-bikes at intersections has been increasing year by year, especially in developing countries.

E-bike accidents are generally attributed to two causes. Firstly, at signalized intersections, e-bike riders exhibit dangerous riding behavior, leading to a much higher violation rate of e-bikes than that of traditional bikes [6]. Secondly, due to the swinging, grouping, and transcendence characteristics of e-bikes [7], the expansion effect is significant when 
crossing an intersection. E-bikes occupy the driving space of motor vehicles at intersections and therefore significantly disturb the normal running of motor vehicles. Moreover, there are many factors that affect the expansion effect of e-bikes. Wei et al. [8] reported that the higher the speed of an e-bike, the greater the transverse distance required. In addition, Tibor et al. [9] found that e-bikes had twice the risk of being involved in traffic accidents in intersections as compared with conventional bikes. According to [10], the number of e-bike accidents in Jiangsu Province accounted for about $70 \%$ of the total traffic accidents from January to June in 2016, especially near irregular intersections. Compared with typical signalized intersections, the traffic flow directions of irregular signalized intersections are irregular [11]. The vehicle driving trajectory is different from that of regular signalized intersections [12]. Therefore, the uncertainty of the expansion effect of e-bikes is more obvious. The expansion effect of e-bikes is the most important release characteristic. Qu et al. [13] studied the factors that influence the expansion effect of straight-going e-bikes at typical intersections and determined the influence degree of different factors on the expansion effect. Chen et al. [14] found that the number and direction of e-bikes arriving during a red light had a significant impact on the dispersion width of left-turn e-bikes. In addition, in intersections, the impact of e-bikes on motor vehicles has also been reflected in the expansion effect of e-bikes. Li et al. [15] reported that e-bike riders usually set out in advance and occupy adjacent roads. These violations have affected the normal passage of motor vehicles. Considering this, most researchers have established simulation models to evaluate the influence of the expansion effect of e-bikes on motor vehicles. For example, a mixed traffic flow signal timing model that considers the advance release of e-bikes has been established. The analysis of Gao et al. [16] showed that when the proportion of e-bikes was $40-60 \%$, the influence of e-bikes on the expansion of motor vehicles could be considered to be in the signal control timing. The cellular automata model, social force model, and particle dispersion model have often been used for the simulation of motor vehicles and e-bikes [17-19]. The influence of e-bikes on motor vehicle traffic has been evaluated by simulating the running track of e-bikes and the interaction characteristics with motor vehicles at a typical signalized intersection.

However, scenarios of irregular signalized intersections have not been considered in previous studies. Since the intersection angle of an irregular signalized intersection is a non-right-angle, the trajectory of traffic flow in each direction deviates and the expansion effect of e-bikes is particularly prominent. The main purpose of this study is to study the expansion effect of straight-going e-bikes at irregular signalized intersections. The specific focus of this study is the flow and spacing of e-bikes during the green light release period at irregular signalized intersections. Furthermore, the concept of expansion degree is introduced based on a clustering method. Finally, the beetle antennae search-back propagation (BAS-BP) neural network model is established to predict the expansion degree of e-bikes going straight at irregular signalized intersections. This study can provide theoretical guidance for the channelization design of irregular signalized intersections and e-bike control.

\section{Experimental Design and Data Analysis}

\subsection{Data Collection}

We select the intersection at Nanjing Longpan Road and Bancang Street and the intersection at Shanghai Road and Huaqiao Road as the research object. Two irregular signalized intersections that are selected have a heavy flow of straight-going e-bikes and a right-turn lane. A Da-Jiang Innovation (DJI) Phantom 4 is used to shoot video at an altitude of about $50 \mathrm{~m}$ above the intersection. Relevant parameters of the unmanned aerial vehicle (UAV) are shown in Table 1. 
Table 1. The specific parameters of the DJI Phantom 4.

\begin{tabular}{|c|c|c|}
\hline Equipment Name & \multicolumn{2}{|c|}{ Specific Technical Parameters } \\
\hline \multirow{6}{*}{ Aircraft } & & Vertical: $\pm 0.1- \pm 0.5 \mathrm{~m}$ \\
\hline & Hover precision & Level: $\pm 0.3- \pm 1.5 \mathrm{~m}$ \\
\hline & Maximum speed of rise & $6 \mathrm{~m} / \mathrm{s}$ \\
\hline & Maximum rate of descent & $4 \mathrm{~m} / \mathrm{s}$ \\
\hline & Maximum horizontal flight speed & $20 \mathrm{~m} / \mathrm{s}$ \\
\hline & Satellite positioning module & GPS/GLONASS \\
\hline \multirow[b]{2}{*}{ Camera } & Pixel & $1 / 2.3$ of an inch CMOS, 12.4 million effective pixels \\
\hline & Shot & FOV94을 $20 \mathrm{~mm}$ (35 mm format equivalent) f/2.8 \\
\hline \multirow{3}{*}{ Cloud platform } & & \\
\hline & Controllable rotation range & Pitch angle $-90^{\circ}-+30^{\circ}$ \\
\hline & Stable system & 3-axis (pitch, roll, and yaw) \\
\hline \multirow{5}{*}{ Other parameters } & Operating temperature & $-10^{\circ} \mathrm{C}-40^{\circ} \mathrm{C}$ \\
\hline & Operating frequency & $2.4 \mathrm{GHz}$ ISM \\
\hline & Cell voltage & $15.2 \mathrm{~V}$ \\
\hline & System version requirements for mobile devices & iOS 8.0 and above \\
\hline & system version requirements ior monile aevices & Android 4.1 .2 and above \\
\hline
\end{tabular}

The survey time is the morning and evening peak hours from 22 December 2020 to 29 and 30 March 2021. The total duration of the survey is $6 \mathrm{~h}$. A total of 97 cycles of green light data are obtained. The geometrical characteristic parameters of the intersections are shown in Figure 1 and Table 2.

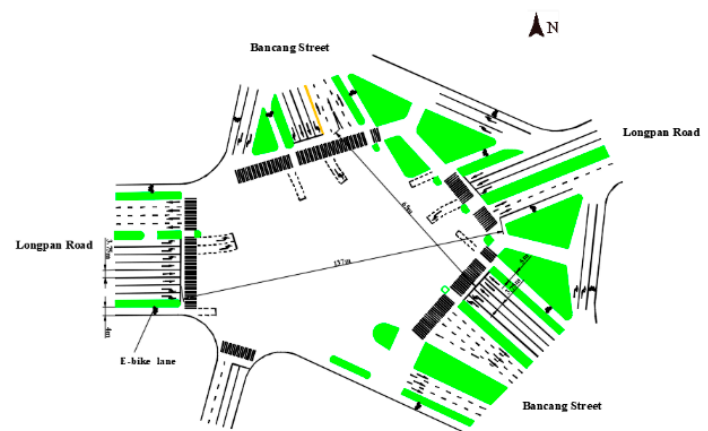

(a)

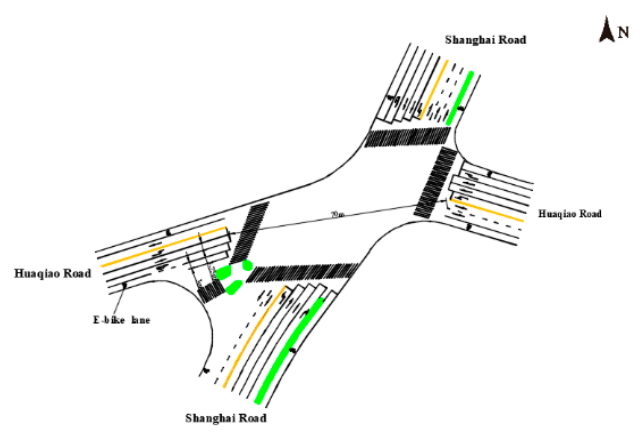

(b)

Figure 1. A diagram of the intersection: (a) the intersection at Longpan Road and Bancang Street; (b) the intersection at Shanghai Road and Huaqiao Road.

Table 2. Survey site-related characteristic parameters.

\begin{tabular}{|c|c|c|c|c|c|c|}
\hline Intersection & $\begin{array}{l}\text { Driving } \\
\text { Directions }\end{array}$ & Survey Time & $\begin{array}{l}\text { The Green } \\
\text { Light Time }\end{array}$ & $\begin{array}{c}\text { Angle of } \\
\text { Declination }\end{array}$ & $\begin{array}{c}\text { Space } \\
\text { Situation }\end{array}$ & $\begin{array}{c}\text { Across } \\
\text { the Street } \\
\text { from }\end{array}$ \\
\hline \multirow{2}{*}{$\begin{array}{l}\text { The intersection of } \\
\text { Longpan Road and } \\
\text { Bancang Street }\end{array}$} & $\begin{array}{l}\text { Northwest to } \\
\text { southeast }\end{array}$ & $\begin{array}{c}22 \text { December 2020, } \\
\text { 7:30-8:30, 17:30-18:30 }\end{array}$ & $38 \mathrm{~s}$ & $19^{\circ}$ & None & $137 \mathrm{~m}$ \\
\hline & $\begin{array}{c}\text { Southwest to } \\
\text { northeast }\end{array}$ & $\begin{array}{c}29 \text { March } 2021 \\
7: 30-8: 30,17: 30-18: 30\end{array}$ & $35 \mathrm{~s}$ & $8^{\circ}$ & Treelawn & $65 \mathrm{~m}$ \\
\hline $\begin{array}{c}\text { The intersection of } \\
\text { Shanghai Road and } \\
\text { Huaqiao Road }\end{array}$ & Southwest to east & $\begin{array}{c}30 \text { March 2021, } \\
7: 30-8: 30,17: 30-18: 30\end{array}$ & $35 \mathrm{~s}$ & $27^{\circ}$ & Rail fence & $79 \mathrm{~m}$ \\
\hline
\end{tabular}

Note: Deflection angle refers to the angle that the driving direction of the intersection deviates from the driving track of straight-going vehicles at the conventional intersection. 
The expansion degree of e-bikes is mainly shown in the lateral offset distance when the vehicle passes an intersection [13]. To facilitate the description of this characteristic, the maximum width of the in-line deviation from the driving direction of an e-bike during green light release was taken as the expansion degree of the e-bike. It is positive when it is toward the motor vehicle space, and negative when it is on the other side.

Next, the video is imported into the Tracker and the coordinate system is set. The $\mathrm{X}$-axis is the middle line of the e-bike from the stop line to the exit road. The Y-axis is perpendicular to the $\mathrm{X}$-axis. The origin is the position of the stop point. Therefore, the change in position parallel to the $X$-axis represents the straight-line travel distance of the e-bike from the stop line to the exit road. The change of position parallel to the Y-axis represents the distance of the e-bike from the center of the road (expansion). The tracker software is used to collect the position coordinate information of each e-bike at every moment (1 s) during the green light release period. A total of 2386 e-bike tracks are collected. Taking the intersection of Longpan Road and Bancang Street as an example, the coordinate system is shown in Figure 2.

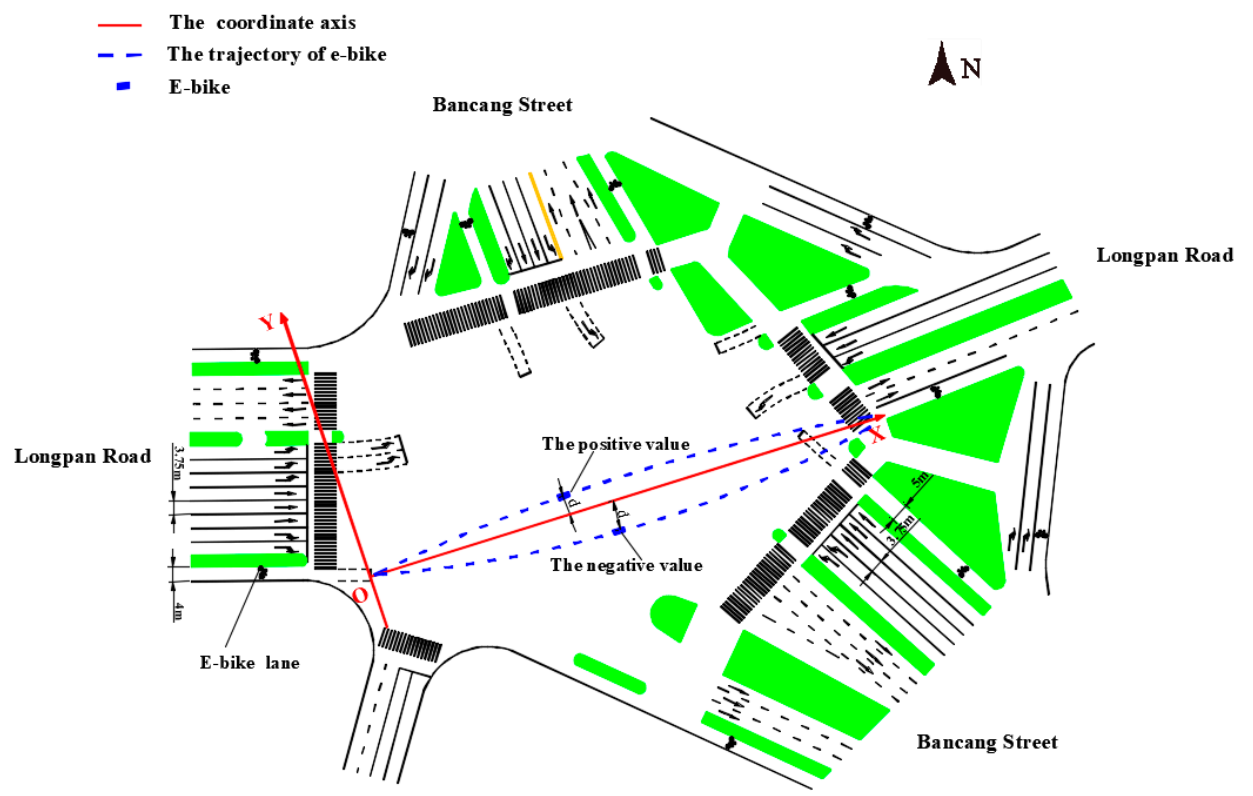

Figure 2. Schematic diagram of the expansion degree of a straight-going e-bike at an irregular signal intersection.

\subsection{Determination of the Release Stage}

Through field observations, it is found that the discharge of e-bikes during the green light release period presents a relatively obvious stage characteristic. Among different release stages, there is a stage in which the degree of expansion changes rapidly (i.e., not every release stage has a significant degree of expansion), which has a great influence on motor vehicle flow. At the same time, in each release stage, the release group of e-bike also presents different changes. Some release groups are always in a large state of expansion when they pass through irregular signalized intersections, while some release groups of e-bikes are almost travelling along the median line. In order to determine the release stage and group with the obvious expansion degree of straight-going e-bikes at irregular signalized intersection, the flow and spacing changes of e-bikes are selected as indicators for comprehensive analysis.

The green cycle duration of the three entry lanes at the irregular signalized intersections is 35,35 , and $38 \mathrm{~s}$, respectively, and $5 \mathrm{~s}$ is used as the time interval. The flow of e-bikes in each time interval during the green light release period of three straight entry lanes is sequentially counted as shown in Figure 3. 


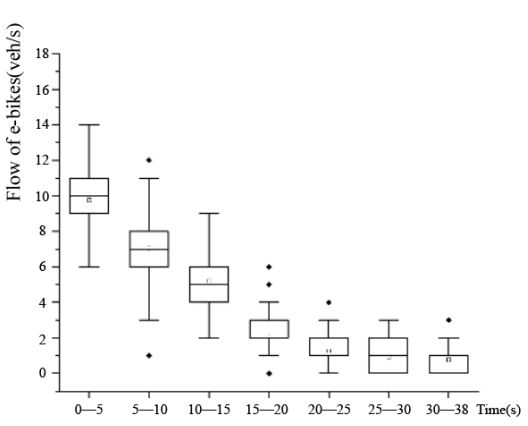

(a)

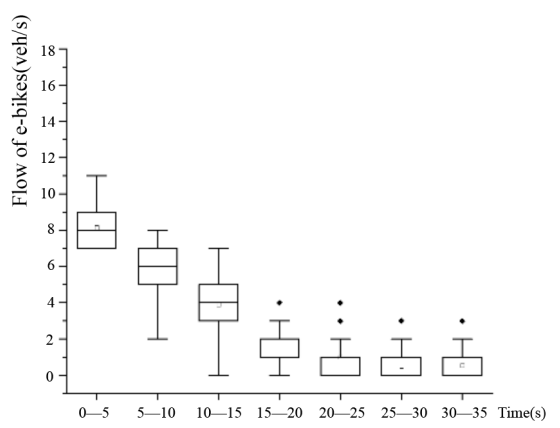

(b)

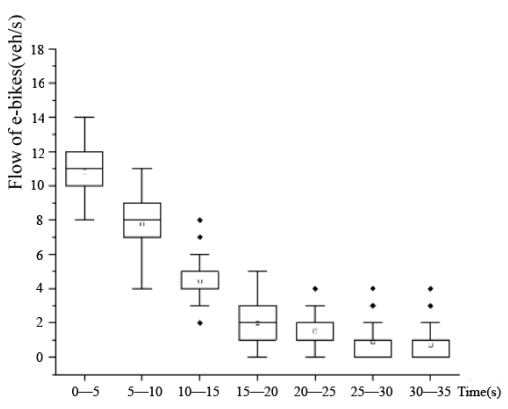

(c)

Figure 3. Straight flow profile of entrance road: (a) Longpan Road and Bancang Street intersection of the northwest entrance road; (b) Longpan Road and Bancang Street intersection of the southwest entrance road; (c) Shanghai Road and Huaqiao Road intersection of the southwest entrance road.

The flow of e-bikes gradually decreases with an increase in time during the seven time intervals of the green light release period. After $15 \mathrm{~s}$, the flow tends to be stable in the time interval. The flow data remained between 2 and 4 veh/s. The flow data of $0-5 \mathrm{~s}$ and 5-10 s are distributed at a higher position than those of other time intervals. Its median value is around $10 \mathrm{veh} / \mathrm{s}$ and can reach as high as $14 \mathrm{veh} / \mathrm{s}$. According to the statistics, the flow and the total flow of the three inlet channels in the time period of $0-15 \mathrm{~s}$ are more than $70 \%$ of all the flow. It can be seen that the discharge of e-bikes during the green light period is mainly concentrated in the first $15 \mathrm{~s}$.

The K-means clustering method is used to further analyze the spacing variation of straight-going e-bikes during the green light release period. The position of the e-bike is drawn in the coordinate system at a time interval of $1 \mathrm{~s}$. The establishment standard of the coordinate system can be seen in Figure 2. Each dot represents an e-bike. The clustering coefficients are $\mathrm{k}=2,3$, and 4 . The change of the spacing of the e-bikes in different clusters at each moment was analyzed, and the stage of straight-going e-bike was the most obvious when $\mathrm{k}=3$. Therefore, straight-going e-bikes are divided into three subgroups to determine the release group with the most obvious expansion degree. We take the spacing change of the green light release period of the northwest entrance road at the intersection of Longpan Road and Bancang Street as an example, as shown in Figure 4.
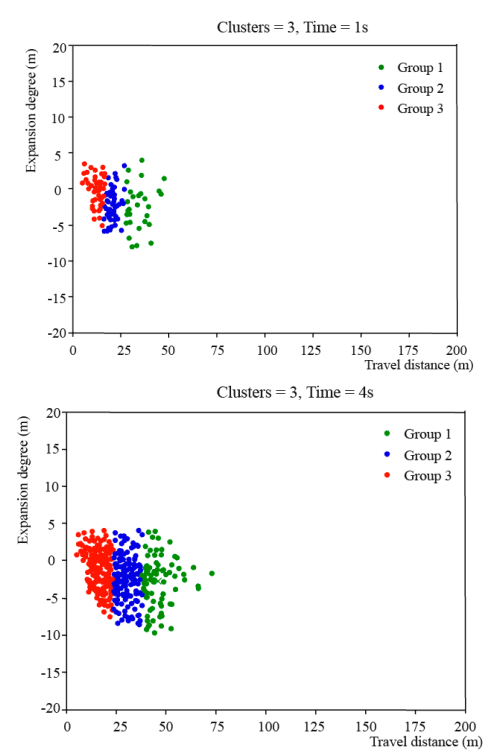
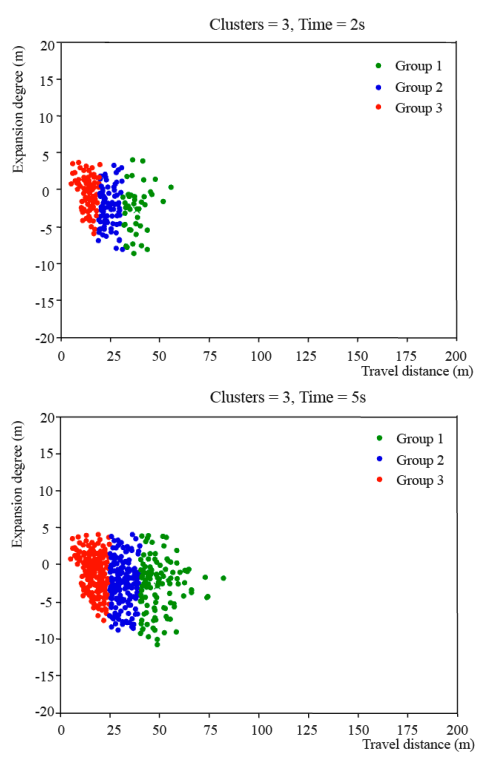

Figure 4. Cont.
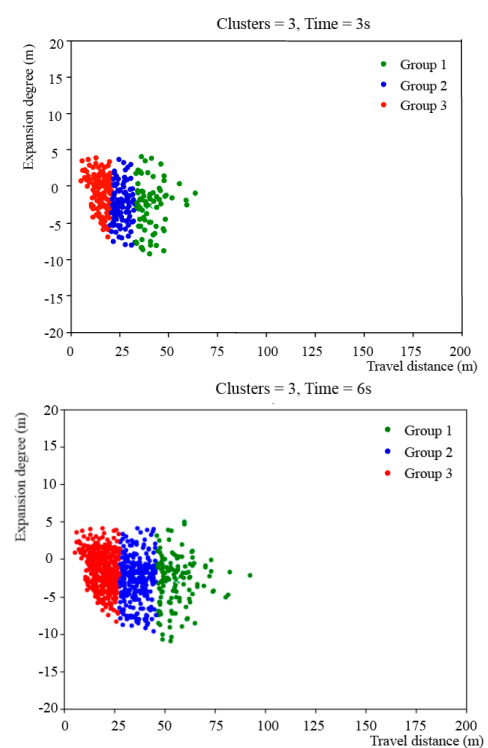

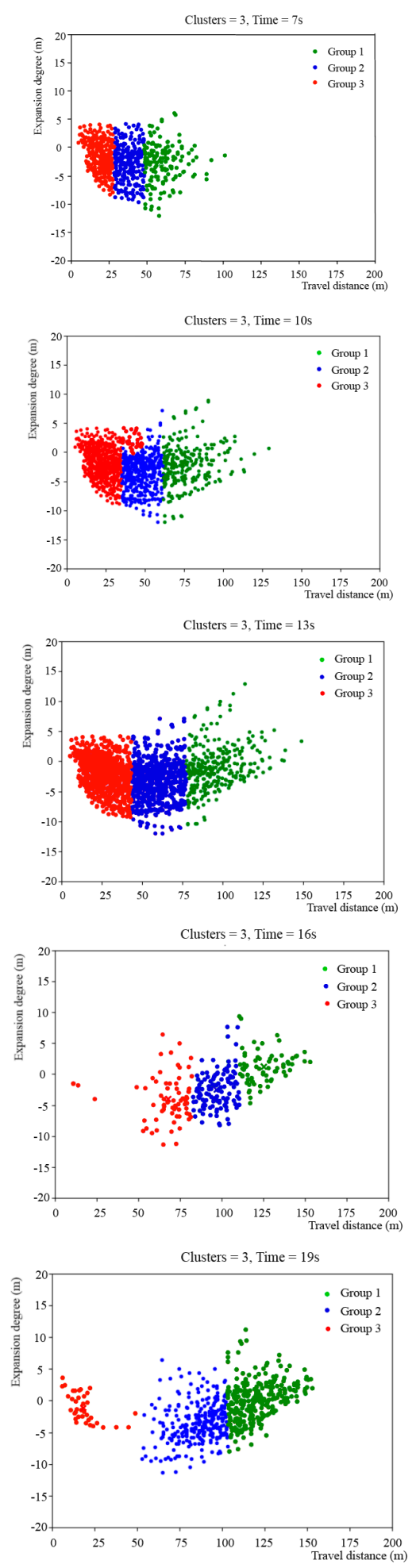
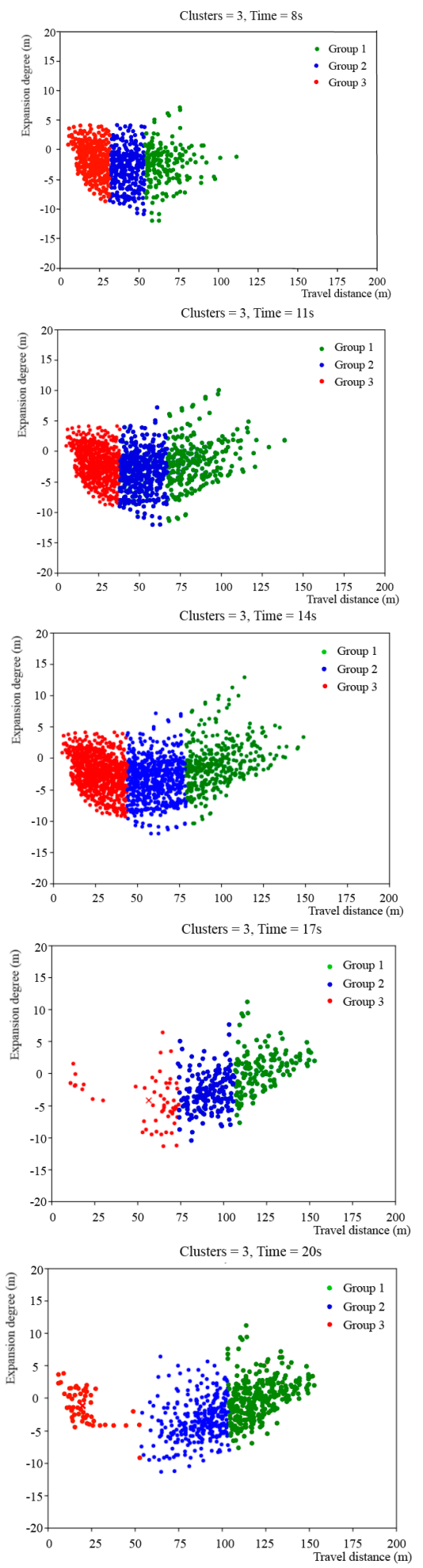
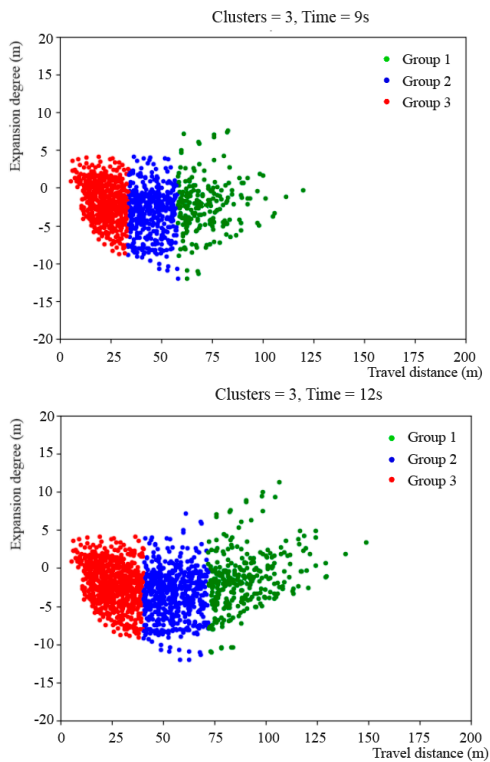

Clusters $=3$, Time $=15 \mathrm{~s}$
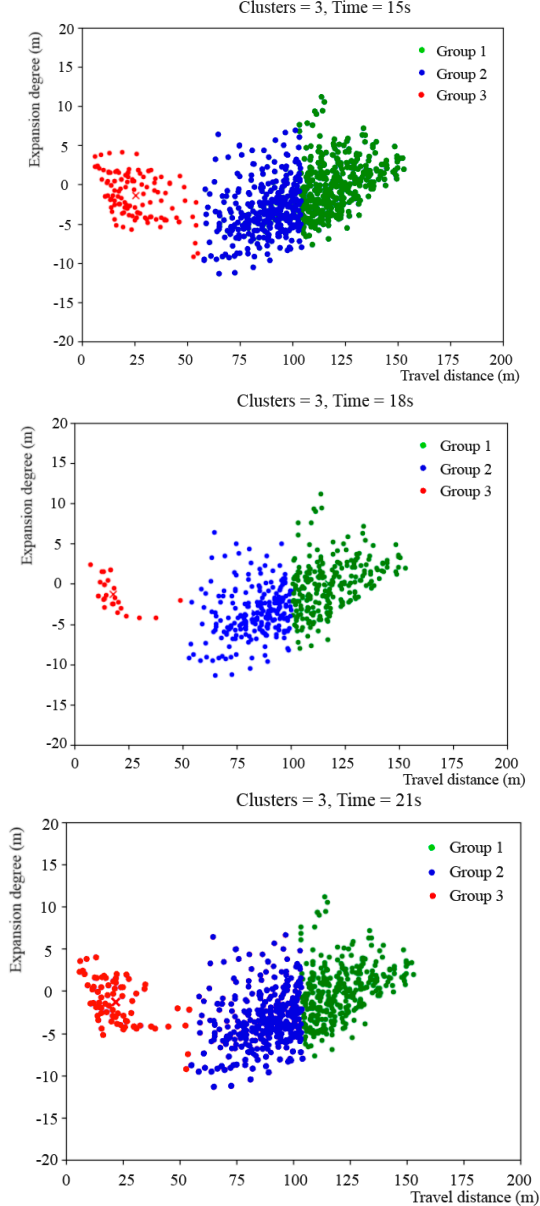

Figure 4. E-bike spacing cluster map for 0-21 s of the northwest entrance road at the intersection of Longpan Road and Bancang Street.

From Figure 4, in 0-4 s, the flow of e-bikes is minimal during the green light release period of the northwest entrance road at the intersection of Longpan Road and Bancang Street. In combination with actual video observations, it may be caused by two factors. On the one hand, some e-bike riders illegally pass beyond the stop line. This will cause them to start moving forward through the intersection when the green lights start. This represents 
a minority of e-bike riders. On the other hand, illegal riders who stop outside the stop line take up the space freed up by queuing vehicles. E-bikes take time to start up. Therefore, only when the front waiting for the e-bike has been passed can the rear line of e-bike pass. In 5-14 s, the flow of the e-bike starts to increase. This is due to the mass of e-bikes in front of the stop line constantly moving forward through the intersection. After $15 \mathrm{~s}$, although the flow of e-bikes gradually increases, the total flow of e-bikes decreases significantly as compared with that of 0-14 s. According to the observation, this is because the first batch of e-bikes that start earlier pass through the intersection in about $15 \mathrm{~s}$. Therefore, this part of the e-bike has been cleared out of the coordinate axis. After the $15 \mathrm{~s}$, e-bikes mainly pass through the intersection in the form of individuals. This conclusion is consistent with the above analysis results that the discharge of e-bikes during the green light period is mainly concentrated in the first $15 \mathrm{~s}$. The time cut-off point of the comprehensive analysis is $14 \mathrm{~s}$. The moment when the flow of e-bikes dropped is used as a node to distinguish the phase of the flow of e-bikes. Thus, the flow of e-bikes can be divided into two stages during the green light release period. Zero to fourteen seconds is the initial stage of release. In this stage, e-bikes show group release and the overall expansion degree is obvious.

Furthermore, the release group category of straight-going e-bike is analyzed. Clustering results show that the position of e-bike in Group 1 has a wide range of expansion in the green light release period and is relatively scattered. This is because Group 1 is released at the early stage, and it quickly occupies the intersection space with the advantage of quick start. The variation range of the expansion degree of Group 2 is relatively concentrated and presents a stable distribution state. Group 2 follows Group 1 closely and is subject to changes in the position of Group 1. In addition, behind Group 2 is Group 3, which is in the state of "front and back attack". Therefore, the expansion degree of Group 2 changes in a small and stable range. The expansion degree of Group 3 shows a smaller change in the form of contraction because Group 3 is mainly composed of individual e-bikes users who do not need to wait for the signal light to pass the intersection directly. At this time, the motor vehicle flow continues to move forward, which limits the change of the expansion degree of Group 3.

Therefore, taking the northwest entrance road of the intersection of Longpan Road and Bancang Street as an example, the Group 1 of e-bikes in the green light release period of $0-14 \mathrm{~s}$ at the intersection is selected as the research object to analyze the expansion degree. Using the same method, it can be determined that $0-15 \mathrm{~s}$ and $0-17 \mathrm{~s}$ are relatively obvious overall expansion degrees of e-bikes at the southwest entrance road of the Longpan Road and Bancang Street intersection and the southwest entrance road of the Shanghai Road and Huaqiao Road intersection. The Group 1 of e-bikes is also taken as the research object with a total of 377 sets of data.

\section{Degree of Expansion of E-Bike}

The beetle antennae search (BAS) algorithm was first proposed in 2017 [20]. It is a new algorithm for multi-objective function optimization based on the longicorn foraging principle, and it can be used to optimize the weight and threshold of a BP neural network to realize the automatic optimization process [21]. Compared with the traditional back propagation (BP) neural network, the BAS-BP neural network optimizes weights and thresholds in the input layer, output layer, and hidden layer structure, which overcomes the problems of poor stability and easily falls into the local optimum of the BP neural network [22]. The diagram of the longicorn beetle and the optimization structure of the BAS-BP neural network were shown in Figures 5 and 6, respectively. The main steps of the algorithm are as follows: 


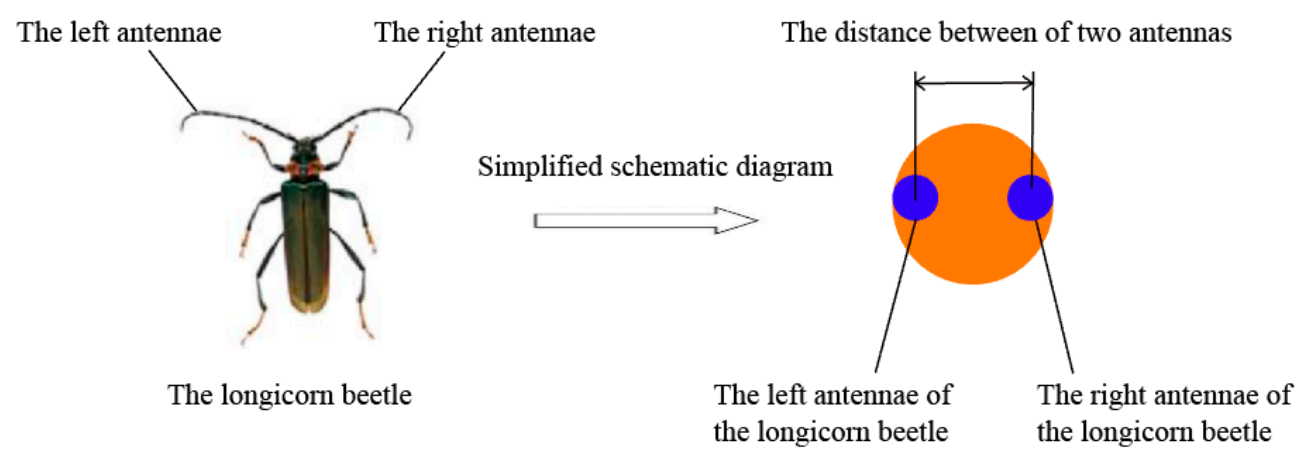

Figure 5. The diagram of the longicorn beetle. (Source: the Baidu website).

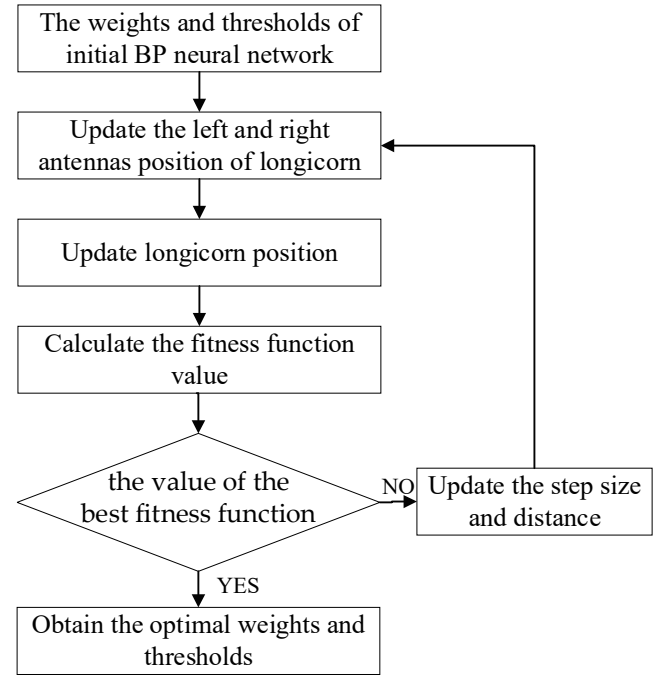

(a)

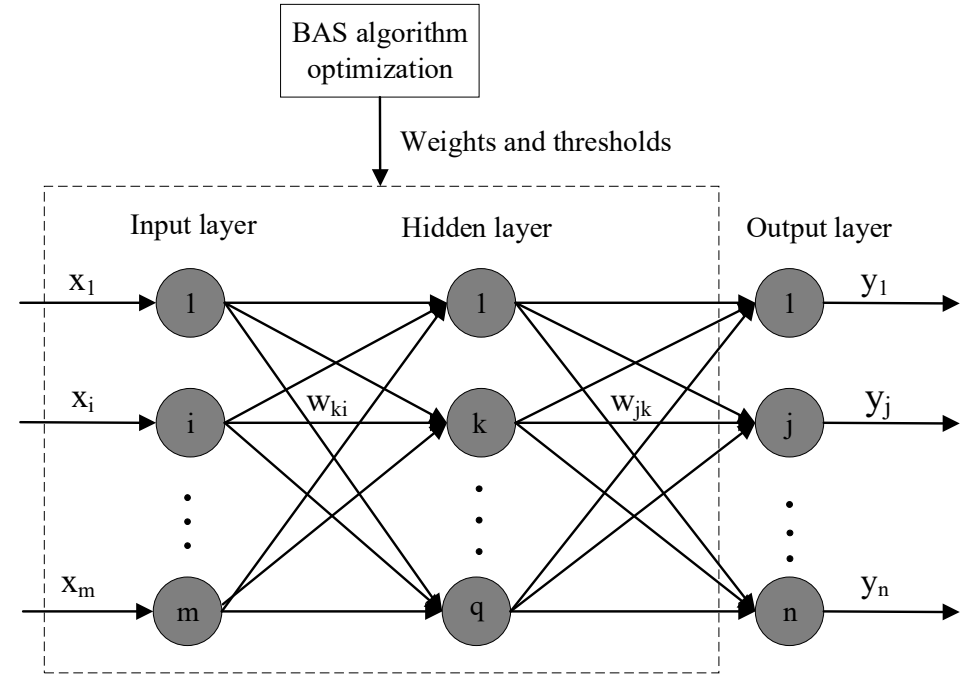

(b)

Figure 6. Schematic diagram of the optimization structure of the BAS-BP neural network: (a) BAS algorithm optimization; (b) neural network structure.

\subsection{Model Establishment}

Step one: The foraging behavior of longhorn beetles is extended to $D$ dimensional space. It can correspond to a $D$ dimensional optimization problem. $D$ represents the number of initial weights and thresholds. The calculation formula of spatial dimension $D$ is shown in Equation (1). We assume that the centroid coordinate of longicorn beetle is $x$; the left and right antennae of longicorn beetle are $x_{l}$ and $x_{r}$, respectively; and the distance between the two antennae is 1 .

$$
D=m \cdot q+q \cdot n+q+n
$$

where $m$ stands for number of neurons in the input layer, $q$ represents the number of hidden layer neurons, and $n$ represents the number of neurons in the output layer.

Step two: The direction of the longicorn beetle is random after each step forwards. The direction of its left and right antennae is also random. The $D$ dimensional unit random vector is established to represent the direction of antennae of longicorn beetle.

$$
\vec{b}=\frac{\operatorname{rands}(D, 1)}{\|\operatorname{rands}(D, 1)\|}
$$

where rands stands for random functions, $D$ represents the spatial dimension of the left and right antennae, and its calculation method is shown in Equation (1). The number "1" means to generate a random vector. 
Step three: Set up left and right antennae spatial coordinates as follows:

$$
\left\{\begin{array}{l}
x_{r t}=x^{t}+l_{0} \cdot \vec{b} / 2 \\
x_{l t}=x^{t}-l_{0} \cdot \vec{b} / 2
\end{array}(t=0,1, \ldots, \mathrm{i})\right.
$$

where $x_{r t}$ is the right antennae coordinate of the th iteration, $x_{l t}$ is the left antennae coordinate of the $t$ th iteration, $x^{t}$ is the centroid coordinate for the $t$ th iteration, and $l_{0}$ is the distance between the two antennae.

Step four: The left and right moves are judged by a fitness function. The mean square error (MSE) of test data is used as the fitness evaluation function to promote a spatial search. It is shown in Equation (4). MSE $E_{l t}$ represents the fitness function value obtained after the algorithm running when the initial weight and threshold value of the solution $t$ are $x_{l t}$ and $M S E_{r t}$ represents the fitness function value obtained after the algorithm running when the initial weight and threshold value of the solution $t$ are $x_{r t} . M S E_{l t}$ and $M S E_{r t}$ are used to represent the fitness function of the left and right antennae of the longhorn beetle for the $t$ th iteration, respectively, to judge the moving position of the longhorn beetle. When the iteration of the algorithm stops, the position where the fitness function value is the smallest is the optimal solution:

$$
M S E=\frac{1}{N} \sum_{i=1}^{N}\left(y_{\text {sim }}^{t}-y^{t}\right)^{2}
$$

where $N$ is the number of samples in the training set, $y_{\text {sim }}^{t}$ is the output value of the $t$ th sample model, and $y^{t}$ is the actual value of the th sample.

Step five: Iterate to update the position of longicorn beetles. If $M S E_{l t}<M S E_{r t}$, longhorn would move to the left of the specified step length. If $M S E_{l t}>M S E_{r t}$, longhorn would move to the right of the specified step length. Therefore, it can be uniformly defined by the sign function in Equation (5)

$$
x^{t+1}=x^{t}-\delta^{t} \cdot \vec{b} \cdot \operatorname{sign}\left(M S E_{r t}-M S E_{l t}\right)
$$

where $\delta^{t}$ is the step size factor at the th iteration and its calculation method is shown in Equation (6)

$$
\delta^{t+1}=\delta^{t} \cdot \beta, t=0,1,2, \ldots, i
$$

where $\beta$ is a value in the range $[0,1]$ and close to 1 . In this paper, $\beta=0.95$. Additionally, the linear decreasing weight strategy is used to determine the initial step size, and the initial step size is 2 .

The step size factor is used to control the searching ability of longhorn beetle. Therefore, the initial step size is selected as large as possible to ensure that the current search area is covered. The calculation formula for step size factor is shown in Equation (6).

Step six: Save the initial centroid coordinates and fitness function values as $X_{\text {best }}$ and $F_{\text {best }}$, respectively. $X_{\text {best }}$ and $F_{\text {best }}$ represent the positions of the best longicorn centroid and the value of the best fitness function. Then, calculate the fitness function value of the current centroid coordinate. If the fitness function value is less than $F_{\text {best }}$, then update $F_{\text {best }}$ and $X_{\text {best. }}$. The algorithm runs until the number of iterations is reached. The value of $X_{\text {best }}$ is substituted into the BP neural network model as the optimal weight and threshold value. In the paper, the number of iterations is set as $i=100$.

The influencing factors of the expansion degree of e-bikes include static factors (related attributes of the intersection) and dynamic factors (motor vehicle flow, e-bike flow, speed) $[23,24]$. Through actual observation, the deviation angle of the intersection with an irregular signal, machine separation setting, and crossing distance are selected as static factors. In addition, the average speed, flow rate, and motor vehicle flow rate of e-bikes are used as dynamic factors to analyze the expansion degree of e-bikes.

On the basis of the influence of dynamic and static factors on the expansion degree of e-bikes, we select six indexes as input variables: the deviation angle of irregular signalized 
intersection, crossing distance, machine separation setting, flow rate of straight-ahead motor vehicles, flow rate of straight-ahead e-bikes, and average speed. Machine non-separation is set as dummy variable, 0 represents inorganic non-separation, 1 represents green belt separation, and 2 represents fence separation. Expansion is taken as the output variable.

The number of hidden layer neurons has a significant influence on the performance of the neural network [25]. When the hidden layer neuron is more than 2, the nonlinear function can be fitted. The relationship between the six variables and the degree of expansion is nonlinear, and the number of neurons in the hidden layer affects the imitative effect. Theoretically, the more neurons in the hidden layer, the stronger the ability to fit the function. However, excessive number of hidden layer neurons will cause the problem of overfitting in practice. Empirical Equation (7) is used to calculate the reasonable range of the number of neurons in the hidden layer. In the neural network model, the neuron of input layer is 6 and the neuron of output layer is 1 . The values of hidden layer neurons ranged from 4 to 12 by Equation (7) (the empirical formula for the number of neurons in the hidden layer, where $\mathrm{a}$ is the regulation parameter). The RMSE value under the number of each neuron is compared successively, and the optimal number of hidden layer neurons is finally selected as 5. The neural network structure is shown in Figure 7.

$$
h=\sqrt{m+n}+a
$$

Here, $h$ represents the number of hidden layer neurons, $m$ represents the number of input layer neurons, $n$ represents the number of neurons in the output layer, and $a$ is a regulation constant between 1 and 10 .

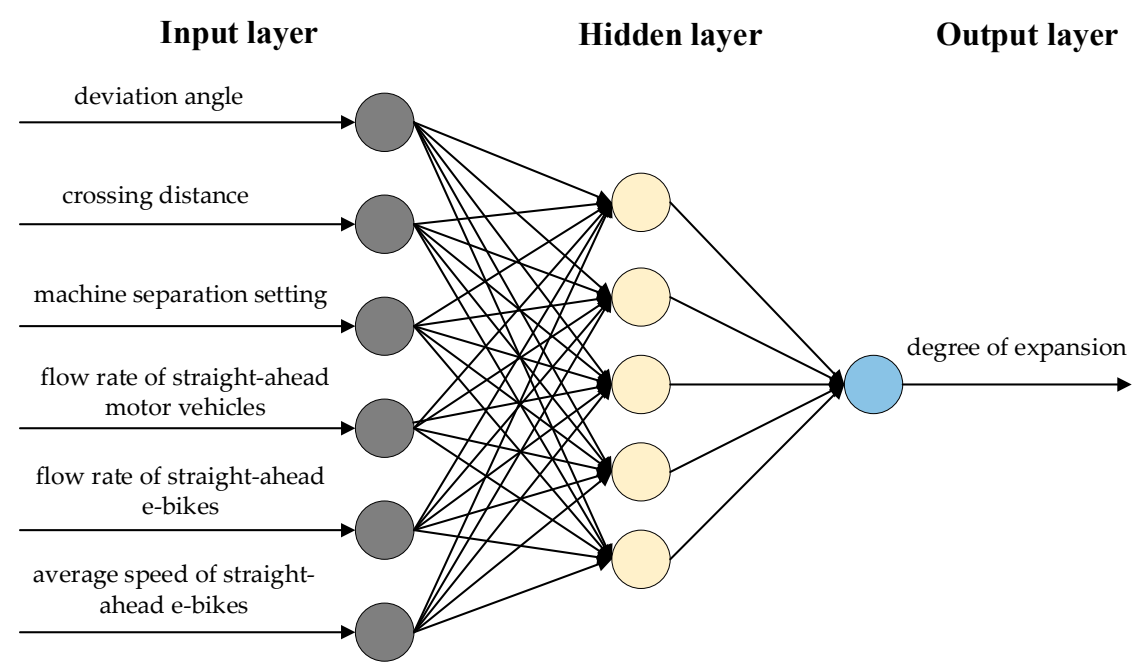

Figure 7. The neural network structure.

The structure of the neural network model is 6-5-1, and the dimension $D$ of search space of the BAS algorithm is 41 (the number of weights is equal to 35 and the number of thresholds is equal to 6). The optimal weights of connected neurons are obtained by BAS algorithm, and 10-times-repeated tests are used to ensure the stability of the model.

\subsection{Model Evaluation}

Two indexes of RMSE and mean relative error (MRE) are selected as the evaluation indexes of the performance of the prediction model. $M R E$ is the ratio of the absolute error of prediction to the true value. The smaller MRE is, the better the model performance is. $R M S E$ can reflect the absolute deviation between the predicted value and the target value and is used to evaluate the degree of change of the model [26]. The calculation formulas are as follows.

$$
M R E=\frac{\left|y_{i}^{\prime}-y_{i}\right|}{y_{i}}(i=1,2, \ldots, N)
$$




$$
R M S E=\sqrt{\frac{1}{n} \sum_{i=1}^{n}\left(y_{i}^{\prime}-y_{i}\right)^{2}}
$$

where $y_{i}$ is the true value and $y_{i}^{\prime}$ is the predicted value.

\subsection{Results and Discussion}

In order to ensure the validity of the model, 377 sets of data are divided into training sets and test sets according to the ratio 7:3 by the set aside method. The code for the BP neural network and the BAS-BP neural network is written and operates by Matlab (version R2014a). The results of 10 repeated runs are used to improve the prediction accuracy. The fitting results of the training and test sets of the BP neural network model and the BAS-BP neural network model are shown in Figures 8 and 9, respectively.
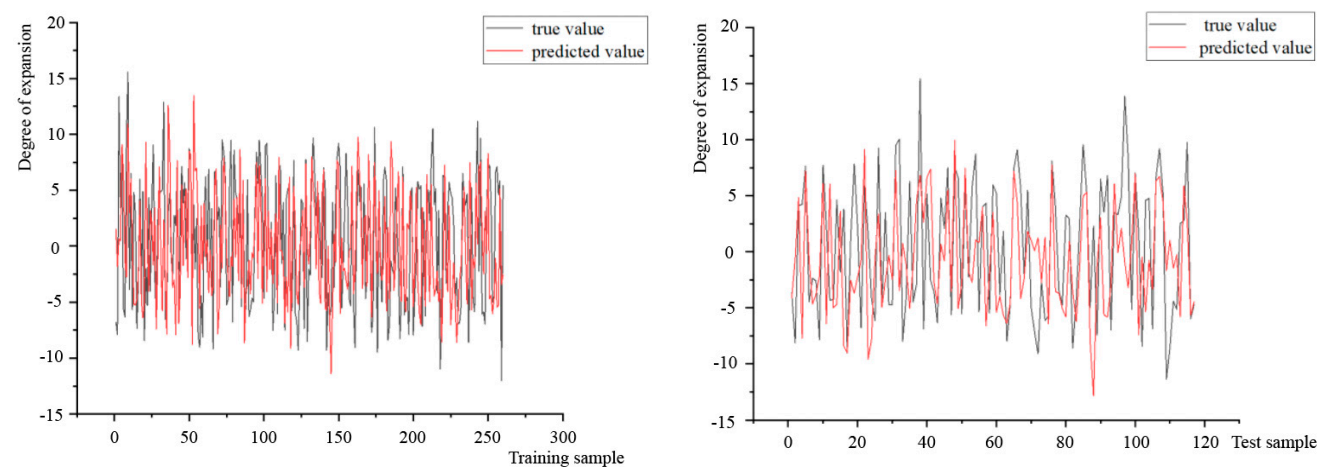

Figure 8. Running results of the BP neural network model.
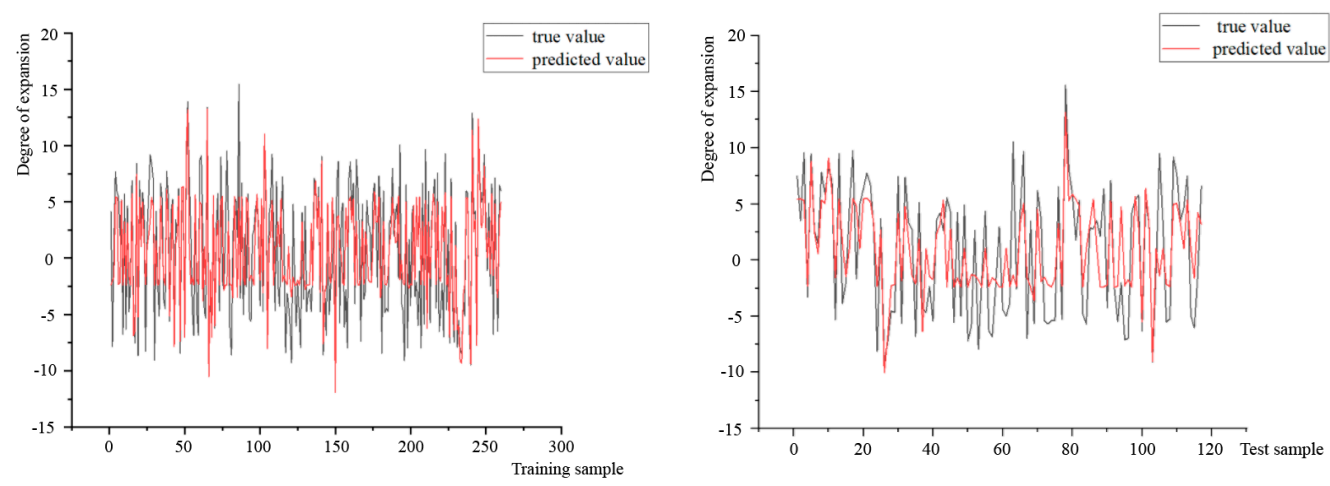

Figure 9. Running results of the BAS-BP neural network model.

According to the running results, the overall trend of the model prediction curve optimized by the BAS algorithm is closer to the real value. Compared with the BP neural network, the difference between the predicted value and the true value of the BAS algorithm optimizes the prediction model curve. Additionally, it has a smaller fluctuation rang. The overall effect is good.

From Table 3, the average relative errors of the training sets of the BP neural network model and the BAS-BP neural network model are 0.95 and 0.76 , respectively. The average relative errors of the test sets are 0.94 and 0.75 , respectively. RMSE values of the training set are 5.27 and 4.22 , respectively. RMSE values of the test sets are 5.60 and 4.38 , respectively. 
Table 3. Evaluation results of the model

\begin{tabular}{ccccccccc}
\hline & \multicolumn{4}{c}{ MRE } & \multicolumn{2}{c}{ RMSE } \\
\cline { 2 - 9 } Times & \multicolumn{2}{c}{ Training Set } & \multicolumn{2}{c}{ Testing Set } & \multicolumn{2}{c}{ Training Set } & \multicolumn{2}{c}{ Testing Set } \\
\cline { 2 - 9 } & BP & BAS-BP & BP & BAS-BP & BP & BAS-BP & BP & BAS-BP \\
\hline 1 & 0.92 & 0.81 & 0.91 & 0.78 & 5.06 & 4.35 & 4.79 & 4.33 \\
2 & 0.89 & 0.73 & 0.82 & 0.6 & 4.73 & 4.22 & 5.74 & 4.43 \\
3 & 1.04 & 0.81 & 0.74 & 0.78 & 5.33 & 4.35 & 5.09 & 4.33 \\
4 & 1.15 & 0.74 & 0.99 & 0.75 & 6.12 & 4.28 & 6.39 & 4.42 \\
5 & 0.83 & 0.77 & 0.94 & 0.79 & 4.89 & 4.3 & 5.28 & 4.36 \\
6 & 0.98 & 0.73 & 0.96 & 0.81 & 5.82 & 4.12 & 6.04 & 4.78 \\
7 & 0.89 & 0.76 & 1.05 & 0.81 & 5.05 & 4.41 & 5.85 & 4.16 \\
8 & 0.96 & 0.8 & 0.99 & 0.74 & 5.26 & 4.43 & 5.97 & 4.49 \\
9 & 0.91 & 0.72 & 1.05 & 0.75 & 5.1 & 3.92 & 5.48 & 4.09 \\
10 & 0.89 & 0.71 & 0.91 & 0.73 & 5.29 & 3.82 & 5.39 & 4.4 \\
AVE. & 0.95 & 0.76 & 0.94 & 0.75 & 5.27 & 4.22 & 5.60 \\
\hline
\end{tabular}

At the same time, according to the variation of fitness curve, the BAS-BP neural network converge faster and reach the MSE optimum at about the 20th iteration. Therefore, as compared with the BP neural network expansion prediction model, the BAS-BP neural network performs better in MRE, RMSE. It shows that the BAS-BP model has good applicability for predicting the expansion degree of e-bikes at irregular signalized intersections. When the expansion degree of straight-going e-bikes at irregular signalized intersections is predicted to be large, traffic organizing strategy (such as advance release of e-bikes) can be optimized. Additionally, channelization layout (such as physical isolation of motor vehicles and e-bikes) can be adjusted. All these measures can reduce the impact of e-bike expansion degree on motor traffic flow.

\section{Conclusions}

In this paper, the expansion degree variation of straight-going e-bikes at irregular signalized intersections was studied. Firstly, the flow characteristics of straight-going ebikes during a green light release period were analyzed. Then, K-means clustering method was used to analyze the spacing variation of the straight-going e-bike. The release stage and release group were determined according to the characteristics of flow and spacing. Finally, a prediction model for the expansion degree of straight-going e-bikes at irregular signalized intersections was proposed based on the BAS-BP neural network. RMSE and $M R E$ were selected to compare the performance of the prediction model before and after BAS algorithm optimization. Conclusions were drawn as follows.

(1) The straight-going e-bike at irregular signalized intersections could be divided into two release stages and three release groups during the green light release period. At the initial stage of release, the flow rate of the e-bike was higher and the expansion degree varies widely. Additionally, the expansion degree of Group 1 changed obviously.

(2) In terms of predicting the expansion degree of straight-going e-bikes at irregular signalized intersections, the prediction model of BAS-BP neural network was obviously better than that of BP neural network. MRE of BAS-BP neural network prediction model and BP neural network prediction model were 0.75 and 0.94 , respectively. The accuracy of $M R E$ was improved by about $25.3 \%$. RMSE of BAS-BP neural network prediction model and BP neural network prediction model were 4.38 and 5.60, respectively. The accuracy of RMSE was improved by about $27.9 \%$.

The expansion degree of straight-going e-bikes at irregular signalized intersections was the research object of this paper. However, the expansion degree of left-turn and right-turn e-bikes at irregular signalized intersections could be studied in the future. 
Author Contributions: All authors contributed substantially to this study. Individual contributions were investigation, T.T. and H.L.; methodology, T.T., J.M. and Z.Y.; and software, M.Z. and C.Z.; and data analysis, T.T.; writing-original draft preparation, T.T., M.Z. and C.Z.; writing-review and editing, J.M. and Z.Y. All authors have read and agreed to the published version of the manuscript.

Funding: Transportation Science and Technology Project of Jiangsu Province, No.2018Y15.

Institutional Review Board Statement: Not applicable.

Informed Consent Statement: Not applicable.

Data Availability Statement: The data are not publicly available due to the institutional restrictions.

Conflicts of Interest: The authors declare no conflict of interest.

\section{References}

1. Forecast Report on Market Competition Pattern and Future Trend of China's E-Bike Industry from 2020 to 2026. Available online: https:/ / www.chyxx.com (accessed on 25 May 2021).

2. Elzbieta, M.; Damian, I. A Back-of-Queue Model of a Signal-Controlled Intersection Approach Developed Based on Analysis of Vehicle Driver Behavior. Energies 2021, 14, 1204.

3. Zhu, Z.; Li, Z.; Liu, Y.; Chen, H.; Zen, J. The impact of urban characteristics and residents' income on commuting in China. Trans. Res. Part D Trans. Environ. 2017, 57, 474-483. [CrossRef]

4. Guo, R.; Liu, J.; Qi, Y. Innovative Signal Timing Strategy for Implementing Contraflow Left-Turn Lanes at Signalized Intersections with Split Phasing. Sustainability 2021, 13, 6307. [CrossRef]

5. Tang, K.; Dong, S.; Wang, F.; Ni, Y.; Sun, J. Behavior of Riders of Electric Bicycles at Onset of Green and Yellow at Signalized Intersections in China. Transp. Res. Rec. J. Transp. Res. Board 2012, 2317, 85-96. [CrossRef]

6. Langford, B.C.; Chen, J.; Cherry, C.R. Risky riding: Naturalistic methods comparing safety behavior from conventional bicycle riders and electric bike riders. Accid. Anal. Prev. 2015, 82, 220-226. [CrossRef]

7. Yu, R.; Zhao, H.; Zhang, C.; Wang, Z. Analysis of risk-taking behaviors of electric bicycle riders in response to pedestrian countdown signal devices. Traffic Inj. Prev. 2019, 20, 182-188. [CrossRef]

8. Wei, L.; Liao, M.; Wang, Z.; Zhuang, S.; Li, Y.; Zhu, J.; Wang, H.; Li, M. Study on Traffic Characteristics and Tolerance Design Method of Turn-left Electric Bicycles Space at Typical Signal Control Intersection. J. Yancheng Inst. Technol. (Nat. Sci. Ed.) 2019, 32, 45-50.

9. Petzoldt, T.; Schleinitz, K.; Heilmann, S.; Gehlert, T. Traffic conflicts and their contextual factors when riding conventional vs. electric bicycles. Transp. Res. Part F Traffic Psychol. Behav. 2016, 46, 477-490. [CrossRef]

10. JSTV.com. Available online: http:/ /news.jstv.com/a/20160805/123632.shtml (accessed on 25 May 2021).

11. Zhang, W.; Wang, B.; Genesis, H. Research on the Improvement Schemes of Irregular Intersection. Appl. Mech. Mater. 2013, 253-255, 1971-1975. [CrossRef]

12. Qin, H.; Guan, H.; Zhao, H.; Long, X.; Zhang, C. Simulation-based Traffic Organization Optimization for Irregular Intersections. J. Transp. Inf. Saf. 2010, 28, 43-48.

13. Qu, Z.; Gao, Y.; Song, X. Effect Analysis of Vehicle-road Environment at Signalized Intersection on the Release and Expansion Characteristics of E-bikes. China J. Highw. Transp. 2020, 33, 126-136.

14. Chen, J.; Wang, W.; Li, Z.; Jiang, H.; Chen, X.; Zhu, S. Dispersion Effect in Left-Turning Bicycle Traffic and Its Influence on Capacity of Left-Turning Vehicles at Signalized Intersections. Transp. Res. Rec. J. Transp. Res. Board 2014, 2468, 38-46. [CrossRef]

15. Li, H.; Zhong, X.; Zhang, W.; Li, S.; Xing, Y. An algorithm for e-bike equivalents at signalized intersections based on traffic conflict events number. Transp. Res. Part A Policy Pract. 2020, 134, 78-95. [CrossRef]

16. Gao, Y.; Qu, Z.; Jiang, J.; Song, X.; Xia, Y. Mixed Traffic Flow Signal Timing Optimization Method Considering E-Bike Expansion Influence. J. Transp. Eng. Part A Syst. 2021, 147, 04020155. [CrossRef]

17. Hang, J.; Lin, Y.; Wang, G.; Wang, D. Evaluation of the dispersion effect in through movement bicycles at signalized intersection via cellular automata simulation. Phys. A Stat. Mech. Its Appl. 2018, 498, 138-147.

18. Yang, D.; Zhou, X.; Wen, C.; Wang, D. Modeling and simulation of motor vehicle-electric bicycle traffic flow at signalized intersection. J. Harbin Inst. Technol. 2018, 50, 181-187.

19. Liu, Q.; Sun, J.; Tian, Y.; Ni, Y.; Yu, S. Modeling and Simulation of Nonmotorized Vehicles' Dispersion at Mixed Flow Intersections. J. Adv. Transp. 2019, 02, 1-19. [CrossRef]

20. Jiang, X.; Li, S. Beetle antennae search without parameter tuning (BAS-WPT) for multi-objective optimization. J. Loss Prev. Process Ind. 2017, 34. [CrossRef]

21. Qian, J.; Wang, P.; Pu, C.; Chen, G. Joint application of multi-object beetle antennae search algorithm and BAS-BP fuel cost forecast network on optimal active power dispatch problems. Knowl. Based Syst. 2021, 226, 107149. [CrossRef]

22. Wang, T.; Liu, Q. The Assessment of Storm Surge Disaster Loss Based on BAS-BP Model. Mar. Environ. Sci. 2018, $37,457-463$.

23. $\mathrm{Xu}, \mathrm{Z}$. Research on Release Characteristics of E-Bikes at Signalized Intersections. Master's Thesis, Jilin University, Changchun, China, 2018 
24. Ding, B.; Song, Z. Analysis of Influencing Factors of Urban Intersections Risks Based on Traffic Conflict. For. Eng. 2019, 35, 98-105.

25. Zhang, D. MATLAB Neural Network Application Design; Machine Press: Beijing, China, 2012.

26. Cao, R.; Zhang, K.; Zeng, W.; Ma, J.; Xie, Y.; Li, Q. Research on the Early-warning Method of Water Environment Carrying Capacity Based on BP Neural Network: A Case Study of Beiyunhe River Basin. Acta Sci. Circumst. 2021, 41, $2005-2017$. 